\title{
High Prevalence of Cyclical Variation in Heart Rate before Nocturnal Episodes of Paroxysmal Atrial Fibrillation
}

\author{
Akira Fujiki, Masao Sakabe and Ryozo Yoshioka
}

\begin{abstract}
Objective Nighttime onset of atrial fibrillation (AF) is sometimes associated with obstructive sleep apnea accompanied by a characteristic heart rate (HR) pattern known as cyclical variation of HR. The aim of this study was to evaluate whether cyclical variation of HR is prevalent in patients with nocturnal AF.

Methods The subjects consisted of 34 patients $(68 \pm 12$ years) with paroxysmal AF, including 14 patients with daytime AF and 20 patients with nighttime AF. Holter electrocardiogram (ECGs) were examined for the presence of cyclical variation in HR and to quantify the HR variability within the 40-minute period preceding each AF episode using a fast Fourier transform (FFT) methods.

Results Cyclical variation in HR was observed in 12 of $20(60 \%)$ nighttime episodes and in only two of 14 (14\%) daytime episodes. The prevalence of cyclical variation in HR was significantly greater in the nighttime $\mathrm{AF}$ episodes than in the daytime AF episodes $(\mathrm{Chi}=5.34, \mathrm{p}<0.05)$. The mean frequency of cyclical variation in HR was $0.015 \pm 0.003 \mathrm{~Hz}$. The mean power of the VLF (very low frequency) component $(0.008-0.04 \mathrm{~Hz}$ ) before the onset of AF was significantly greater in the nighttime AF episodes than in the daytime AF episodes. Among the nighttime AF episodes, the power of the HF (high frequency), LF (low frequency) and very low frequency (VLF) components increased significantly just before the onset of AF compared with that observed 40 minutes before onset.

Conclusion The high prevalence of cyclical variation in HR observed before nocturnal AF episodes suggests that sleep apnea may play a role in the onset of nighttime AF.
\end{abstract}

Key words: atrial fibrillation, heart rate variability, fast fourier transformation, sleep apnea

(Intern Med 52: 2169-2172, 2013)

(DOI: 10.2169/internalmedicine.52.0787)

\section{Introduction}

An association between nocturnal atrial fibrillation (AF) and sleep apnea has been suggested by several investigators $(1,2)$. In patients with obstructive sleep apnea under 65 years of age, the cumulative frequency of AF is significantly increased compared with that observed in patients without sleep apnea (3). However, direct evidence supporting sleep apnea as a trigger of AF is lacking. During sleep studies, it is sometimes difficult to record both sleep apnea and $\mathrm{AF}$ episodes simultaneously, because the frequency of nocturnal AF events is not high. On the other hand, using Holter monitoring, episodes of AF can be recognized frequently. Guilleminault et al. demonstrated cyclical variations in heart rate (HR) during sleep apnea episodes (4). Stein et al. proposed a simple method for identifying sleep apnea using cyclical variation in $\mathrm{HR}$ on Holter electrocardiogram (ECG) (5). The aim of this study was to evaluate whether cyclical variation in HR is prevalent in patients with nocturnal AF. AF with a nocturnal occurrence is expected to be associated with sleep apnea that exhibits a cyclical variation in HR.

\section{Materials and Methods}

This study consisted of 34 patients $(68 \pm 12$ years, 28 men and six women) with paroxysmal AF, including 14 patients with daytime onset and 20 patients with nighttime onset. The mean left ventricular ejection fraction of the patients 


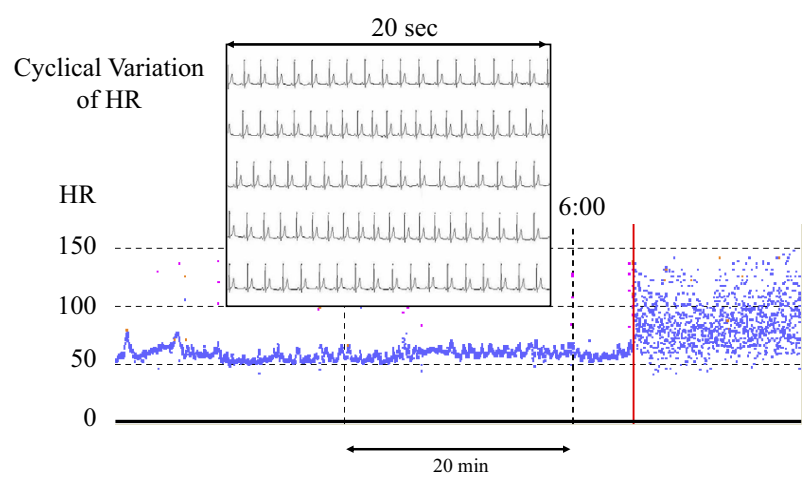

Figure 1. Cyclical variation in the heart rate (HR) before the onset of nocturnal atrial fibrillation (AF). A 70-year-old man had nocturnal paroxysmal AF episodes.The trend in HR at the onset of AF (red line) occurring during sleep in the early morning (6 am) revealed regular cyclical variation in HR. The amplitude was $>20$ beats and the duration was $>10$ minutes. The frequency of cyclical variation in HR was approximately 0.018 Hz. The ECG exhibits cyclical variation in HR.

was $0.60 \pm 0.16$. The patients were excluded if they had a preceding history of coronary artery disease, valvular heart disease or cardiomyopathy. Any patients with structural heart disease diagnosed based on noninvasive methods, including echocardiography, were also excluded. Digital Holter ECG recording devices (FM-180, Fukuda Denshi, Japan) with a sampling rate of $128 / \mathrm{sec}$ were used with an automatic measurement system (SCM-6600, Fukuda Denshi, Japan). Daytime and nighttime was determined in each patient as the awake and sleep states, respectively, assessed according to the 24-hour Holter ECG recordings. The Holter ECGs were examined for the presence of cyclical variation in $\mathrm{HR}$ occurring within the 40-minute period preceding each AF episode. Cyclical variation in $\mathrm{HR}$ was defined as an HR change of $>20$ beats/minute per cycle with a duration of $>10$ minutes (5). Frequency domain measurements of HR variability were also determined in each segment preceding AF using a fast Fourier transform (FFT) analysis. In the FFT analysis, premature atrial and ventricular contractions were excluded; only normal RR intervals were included. The 2,560-second interval before the onset of AF was divided into five segments of 512 seconds, and a frequency analysis was applied to each 512-second segment. The power spectra were quantified by measuring the area in three frequency bands: low frequency (LF) power from 0.04 to $0.15 \mathrm{~Hz}$, high frequency (HF) power from 0.15 to $0.40 \mathrm{~Hz}$ and very low frequency (VLF) power from 0.008 to $0.04 \mathrm{~Hz}$. A correlation between VLF power and the cyclical variation in HR caused by sleep apnea has been reported $(6,7)$. The ratio of $\mathrm{LF}$ to $\mathrm{HF}(\mathrm{L} / \mathrm{H})$ was calculated as the index of the sympathetic activity. To evaluate the RR interval fluctuations caused by each frequency component relative to the mean $\mathrm{RR}$ interval, the coefficient of component variance (CCV) (calculated according to the following equation: $\operatorname{CCV}(\%)=$ $100 *$ (power of component $)^{1 / 2} /($ mean RR interval)) was meas-

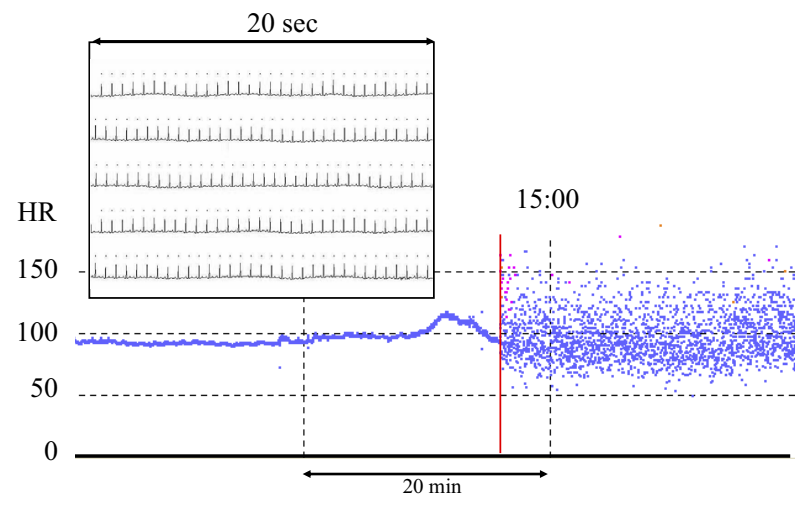

Figure 2. No cyclical variation in heart rate (HR) before the onset of daytime atrial fibrillation (AF). A 63-year-old man had daytime onset of paroxysmal AF episodes (red line) occurring in the afternoon. Before onset, his HR increased to 120 beats/minute, and no regular cyclical variation in HR was observed.

ured (8).

The results are presented as the mean \pm standard deviation (SD). Paired and unpaired data were analyzed using Student's $t$-test. Serial changes in the indices of HR variability were analyzed using an analysis of variance for repeated measures. Statistical significance was set at $\mathrm{p}<0.05$.

\section{Results}

A representative cyclical variation in HR just before the onset of nocturnal AF is shown in Fig. 1. The cyclical variation in HR increased along the time course preceding the nighttime onset of AF. On the other hand, as shown in Fig. 2, the HR increased to 120 beats/minute, and no cyclical variation in HR was observed before the daytime onset of AF. During the 40-minute period prior to AF onset, cyclical variation in HR was observed in 12 of 20 nighttime AF episodes and two of 14 daytime AF episodes. Cyclical variation in HR was significantly more prevalent in the nighttime AF episodes than in the daytime AF episodes (Chi= $5.34, \mathrm{p}<0.05)$. The mean frequency of the cyclical variation in $\mathrm{HR}$ was $0.015 \mathrm{~Hz}$, which was located within the power of the VLF component in the FFT analysis.

The mean power of each frequency component within the 40-minute period preceding each AF episode was compared between the patients with daytime and nighttime onset of paroxysmal AF (Fig. 3). Only the VLF component in the patients with nighttime AF was significantly greater than that observed in the patients with daytime AF. There were no significant differences in LF, HF or L/H between the patients with daytime and nighttime AF. Among the patients with daytime AF, there were no significant differences in the power of VLF, LF and HF or the L/H ratio between the periods just before and 40 minutes before the onset of AF. However, among the patients with nocturnal AF, the power of the VLF, LF and HF components increased significantly just before the onset of AF compared with that observed 40 


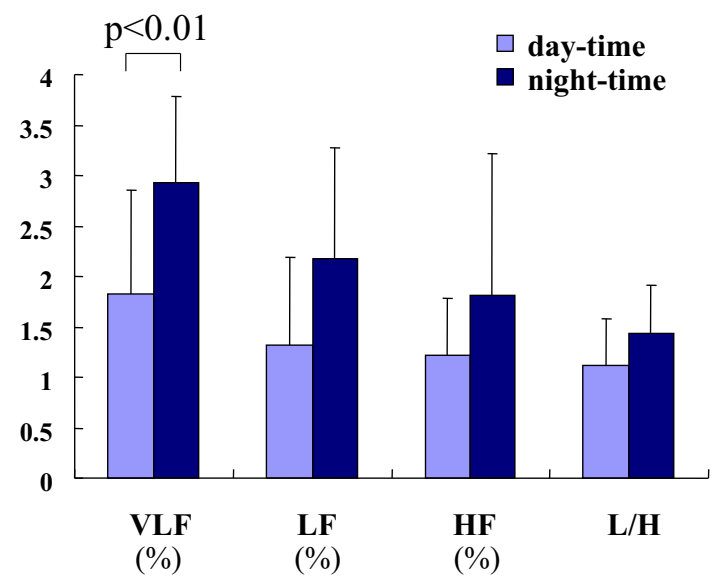

Figure 3. Differences in the mean very low frequency (VLF), low frequency (LF), high frequency (HF) and L/H within the 40-minute period preceding atrial fibrillation (AF) onset between the episodes of daytime and nighttime onset of AF. The mean power of each frequency component within the 40-minute period was compared between the patients with daytime and nighttime onset of paroxysmal AF. Only the VLF component in the patients with nighttime AF was significantly greater than that observed in the patients with daytime AF. There were no significant differences in LF, HF or L/H between the daytime and nighttime AF patients.

minutes before the onset of AF (Fig. 4).

\section{Discussion}

The major findings of the present study are as follows: (1) the prevalence of cyclical variation in HR was significantly greater in the patients with nighttime onset AF than in the patients with daytime onset AF; and (2) the mean power of the VLF component before the onset of AF was significantly greater in the nighttime AF episodes than in the daytime AF episodes. In the nighttime AF episodes, the power of the VLF component increased significantly just before the onset of AF compared with that observed $40 \mathrm{~min}-$ utes before the onset of AF. These observations suggest that sleep apnea plays a role in the onset of nocturnal AF.

An association between nocturnal $\mathrm{AF}$ and sleep apnea syndrome has been reported by several investigators $(1,2)$. Stevenson et al. compared AF patients with controls and found that the proportion of patients with at least moderate obstructive sleep apnea was significantly higher in the AF group than in the control group (62 vs. 38\%) (9). Sleep apnea syndrome is also known to be a risk factor for the recurrence of AF following electrical cardioversion. Kanagala et al. demonstrated that the rate of recurrence of AF in patients with untreated sleep apnea was significantly higher than that observed in patients with treated sleep apnea (10).

A case of paroxysmal AF occurring after a prolonged apnea episode during polysomnography was recently reported (11). Monahan et al. demonstrated that the relative risk of nocturnal paroxysmal AF is increased after sleep- disordered breathing events, although the absolute arrhythmia rate is low (12). Their findings suggested a direct temporal link between sleep apnea events and the onset of AF. We demonstrated that cyclical variation in HR is more frequently observed before nocturnal AF onset than daytime AF onset. The findings of the present study also support the existence of a close relationship between preceding sleep apnea and the nocturnal onset of paroxysmal AF.

Guilleminault et al. first demonstrated the cyclical variation in HR during sleep apnea episodes (bradycardia during apnea followed by tachycardia on termination of apnea) (4). Shiomi et al. reported that the VLF band of the FFT analysis of HR variability is useful for identifying cyclical variations in HR caused by sleep apnea (6). Subsequently, Roche et al. demonstrated that the VLF component of the FFT analysis represents an efficient tool for screening obstructive sleep apnea (7). Stein et al. demonstrated that the presence of cyclical variation in HR with a higher amplitude ( $>20$ beats/minute per cycle) and longer duration ( $>20 \%$ of 3-hour observation period) strongly suggests the presence of sleep apnea (5). Recently, Hayano et al. developed a new automated ECG algorithm to detect obstructive sleep apnea (13). This new algorithm exhibits high sensitivity and specificity for identifying obstructive sleep apnea.

Herweg et al. reported that the HF component is increased preceding nocturnal AF episodes in patients with structurally normal hearts (14). In the present study, the HF, LF and VLF components each significantly increased before the onset of nighttime AF episodes. The HF component reflects the vagal tone, while the LF component indicates sympathovagal balance (15). The VLF component is more complex and reflects temperature regulation and the activity of the renin-angiotensin system in addition to sleep apnea episodes (16). Hence, dynamic changes in autonomic balance in association with sleep apnea episodes may play a role in the initiation of nighttime onset of AF. Several underlying mechanisms of the induction of AF by sleep apnea episodes have been proposed, including sympathovagal imbalance and hypoxemia (17). Although the precise mechanisms remain unknown, some studies have reported the usefulness of continuous positive airway pressure therapy in suppressing nocturnal paroxysmal AF (18).

This study was performed in a limited number of patients who had either daytime or nighttime onset of AF episodes. Differences in autonomic nerve activity between daytime and nighttime onset of AF may affect HR variability differently. Because this study was based on Holter ECG recordings, most of the patients with nighttime onset AF were not evaluated with polysomnography; therefore, the presence of sleep apnea was not confirmed directly using sleep studies.

\section{Conclusion}

The high prevalence of cyclical variation in HR before nocturnal AF episodes suggests that sleep apnea plays a role in the onset of nocturnal AF. 


\section{Day-time AF}

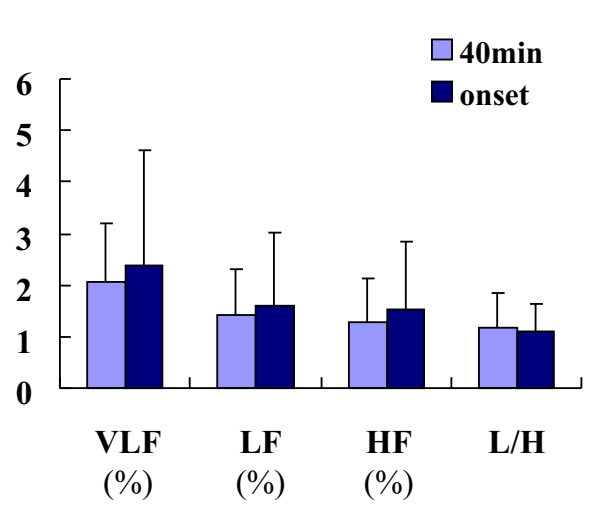

Night-time AF

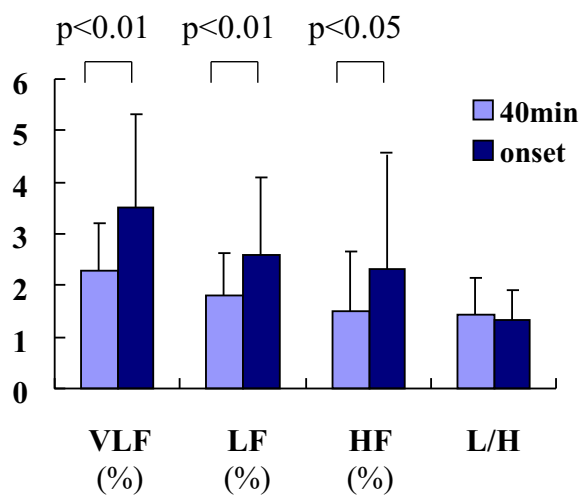

Figure 4. Changes in the very low frequency (VLF), low frequency (LF), high frequency (HF) and L/H 40 minutes before and just before the onset of atrial fibrillation (AF) in the patients with daytime and nighttime AF. In order to evaluate time series changes before the onset of paroxysmal AF, we compared the VLF, LF, HF and L/H 40 minutes before and just before the onset of AF. Among the daytime AF patients, there were no differences in these values. However, among the nocturnal AF patients, the power of the VLF, LF and HF components was increased significantly just before the onset of AF compared with that observed 40 minutes before the onset of $\mathrm{AF}$.

The authors state that they have no Conflict of Interest (COI).

\section{References}

1. Gami AS, Pressman G, Caples SM, et al. Association of atrial fibrillation and obstructive sleep apnea. Circulation 110: 364-367, 2004.

2. Konecny T, Brady PA, Orban M, et al. Interactions between sleep disordered breathing and atrial fibrillation in patients with hypertrophic cardiomyopathy. Am J Cardiol 105: 1597-1602, 2010.

3. Gami AS, Hodge DO, Herges RM, et al. Obstructive sleep apnea, obesity, and the risk of incident atrial fibrillation. J Am Coll Cardiol 49: 567-571, 2007.

4. Guilleminault C, Connolly S, Winkle R, Melvin K, Tilkian A. Cyclical variation of the heart rate in sleep apnoea syndrome. Mechanisms and usefulness of $24 \mathrm{~h}$ electrocardiography as a screening technique. Lancet 21: 126-131, 1984.

5. Stein PK, Duntley SP, Domitrovich PP, Nishith P, Carney RM. A simple method to identify sleep apnea using Holter recordings. J Cardiovasc Electrophysiol 14: 467-473, 2003.

6. Shiomi T, Guilleminault C, Sasanabe R, Hirota I, Maekawa M, Kobayashi T. Augmented very low frequency component of heart rate variability during obstructive sleep apnea. Sleep 19: 370-377, 1996.

7. Roche F, Duverney D, Court-Fortune I, et al. Cardiac interbeat interval increment for the identification of obstructive sleep apnea. Pacing Clin Electrophysiol 25: 1192-1199, 2002.

8. Hayano J, Sakakibara Y, Yamada M, et al. Decreased magnitude of heart rate spectral components in coronary artery disease. Its relation to angiographic severity. Circulation 81: 1217-1224, 1990.

9. Stevenson IH, Teichtahl H, Cunnington D, Ciavarella S, Gordon I, Kalman JM. Prevalence of sleep disordered breathing in paroxys- mal and persistent atrial fibrillation patients with normal left ventricular function. Eur Heart J 29: 1662-1669, 2008.

10. Kanagala R, Murali NS, Friedman PA, et al. Obstructive sleep apnea and the recurrence of atrial fibrillation. Circulation 107: 25892594, 2003.

11. Schultz R, Eisele HJ, Seeger W. Nocternal atrial fibrillation in a patient with obstructive sleep apnea. Thorax 60: 174, 2005.

12. Monahan K, Storfer-Isser A, Mehra R, et al. Triggering of nocturnal arrhythmias by sleep-disordered breathing events. J Am Coll Cardiol 54: 1797-1804, 2009.

13. Hayano J, Watanabe E, Saito Y, et al. Screening for obstructive sleep apnea by cyclic variation of heart rate. Circ Arrhythm Electrophysiol 4: 64-72, 2011.

14. Herweg B, Dalal P, Nagy B, Schweitzer P. Power spectral analysis of heart period variability of preceding sinus rhythm before initiation of paroxysmal atrial fibrillation. Am J Cardiol 82: 869-874, 1998.

15. Hayashi H, Fujiki A, Tani M, Mizumaki K, Shimono M, Inoue H. Role of sympathovagal balance in the initiation of idiopathic ventricular tachycardia originating from right ventricular outflow tract. Pacing Clin Electrophysiol 20: 2371-2377, 1997.

16. Bigger JT Jr, Fleiss JL, Steinman RC, Rolnitzky LM, Kleiger RE, Rottman JN. Frequency domain measures of heart period variability and mortality after myocardial infarction. Circulation 85: 164171, 1992.

17. Javaheri S, Corbett WS. Association of low $\mathrm{PaCO} 2$ with central sleep apnea and ventricular arrhythmias in ambulatory patients with stable heart failure. Ann Intern Med 128: 204-207, 1998.

18. Harbison J, O'Reilly P, McNicholas WT. Cardiac rhythm disturbances in the obstructive sleep apnea syndrome: effects of nasal continuous positive airway pressure therapy. Chest 118: 591-595, 2000 .

(C) 2013 The Japanese Society of Internal Medicine http://www.naika.or.jp/imonline/index.html 